\title{
Concise Commentary: Controversial Interaction of Interleukin-17 with Intestinal Fibrosis
}

\author{
Giovanni Latella ${ }^{1}$
}

Published online: 27 February 2020

(c) Springer Science+Business Media, LLC, part of Springer Nature 2020

Intestinal fibrosis is a common consequence of inflammatory bowel diseases (IBD), especially in patients with Crohn's disease. Fibrosis is generally complicated by intestinal stricture and even obstruction, requiring surgery in $30-40 \%$ of cases. Effective pharmacological treatments aimed at controlling the progression of the intestinal fibrotic process are unavailable.

Fibrosis is characterized by excessive local deposition of extracellular matrix (ECM) proteins, due to their increased production by activated myofibroblasts and/or their reduced degradation by specific matrix metalloproteinases (MMPs). Initiation and progression of fibrosis are modulated by several pro- and anti-fibrogenic molecules, also including the interleukin (IL)-17 family that consists of six related proteins: IL-17A (IL-17), IL-17B, IL-17C, IL-17D, IL-17E, and IL-17F, primarily produced by Th17 cells [1]. These IL-17 cytokines have distinctive effects on IBD; furthermore, their precise contribution to the development of intestinal fibrosis remains unclear.

In this issue of Digestive Diseases and Sciences, Li et al. [2] evaluated how IL-17 affects the development of 2,4,6-trinitrobenzene sulfonic acid (TNBS)-induced chronic colitis-associated fibrosis in mice and the effect of anti-IL-17 treatment on colorectal fibrosis. Serum levels of IL-17, IL- $1 \beta$, transforming growth factor $\beta 1$ (TGF- $\beta 1$ ), and tumor necrosis factor- $\alpha$ (TNF- $\alpha$ ) were all upregulated in TNBS-induced colitis-associated fibrosis and decreased after treatment with IL-17 antibody compared with control TNBS colitis mice treated with IgG. IL-17 antibody-treated TNBS mice had reduced colitis activity, fibrosis, and colorectal mRNA and protein expression of collagen 3, IL-17,

Giovanni Latella

giolatel@tin.it

1 Gastroenterology Unit, Department of Life, Health and Environmental Sciences, University of L'Aquila, Piazza

S. Tommasi n.1, Coppito, 67100 L'Aquila, Italy
TNF- $\alpha$, inhibitor of metalloproteinases- 1 , and MMP-2 compared with controls.

Although these data suggest that IL-17 is involved in the development of colorectal fibrosis, data regarding the dose-response of the anti-IL17 treatment are lacking, as well as the effects on other pro-inflammatory and pro-fibrotic cytokines not suppressed by IL-17 antibodies. It is unclear, though, whether the treatment with IL-17 antibodies has a specific and direct anti-fibrotic effect or whether its antifibrotic effect is secondary to its anti-inflammatory action. Furthermore, the effects of IL-17 antibody treatment were only partial, suggesting that other important factors are involved in the pathogenesis of intestinal fibrosis.

In addition to being involved in the inflammatory and fibrogenic process, the IL-17 family and IL-17-related cytokines are also involved in the gut epithelial homeostasis and the maintenance of mucosal barrier function. Studies reported that blocking of IL-17 in vivo, both through treatment with IL-17 monoclonal antibodies and using IL-17 knockout (KO) mice, increased susceptibility to colitis (as well as severity) in different experimental colitis models (TNBS- and DSS-induced colitis, CD45RBhi transfer colitis) [3-6]. Furthermore, two clinical trials showed that IL-17 blockade had no significant efficacy in patients with Crohn's disease, even increasing the disease activity $[7,8]$. All of these results demonstrate a debatable involvement of IL-17 in intestinal inflammation and fibrosis, requiring further studies to better define its involvement.

An improved knowledge of how IL-17 influences chronic inflammation (as in IBD) could be beneficial in designing new therapeutic approaches in the prevention and treatment of intestinal fibrosis.

Acknowledgements No financial support was received for the study. 


\section{Compliance with Ethical Standards}

Conflict of interest The author has no conflict of interest to declare and did not use any outside assistance in preparing the manuscript.

\section{References}

1. Lawrance IC, Rogler G, Bamias G, et al. Cellular and molecular mediators of intestinal fibrosis. J Crohns Colitis. 2017;11:1491-1503.

2. Li J, Liu L, Zhao Q, Chen M. Role of interleukin-17 in pathogenesis of intestinal fibrosis in mice. Dig Dis Sci. (Epub ahead of print). https://doi.org/10.1007/s10620-019-05969-w.

3. Ogawa A, Andoh A, Araki Y, Bamba T, Fujiyama Y. Neutralization of interleukin-17 aggravates dextran sulfate sodium-induced colitis in mice. Clin Immunol. 2004;110:55-62.

4. Zhang HJ, Xu B, Wang H, et al. IL-17 is a protection effector against the adherent-invasive Escherichia coli in murine colitis. Mol Immunol. 2018;93:166-172.
5. Zhang Z, Zheng M, Bindas J, Schwarzenberger P, Kolls JK. Critical role of IL-17 receptor signaling in acute TNBS-induced colitis. Inflamm Bowel Dis. 2006;12:382-388.

6. O'Connor W, Kamanaka M, Booth CJ, et al. A protective function for interleukin 17A in $\mathrm{T}$ cell-mediated intestinal inflammation. Nat Immunol. 2009;10:603-609.

7. Hueber W, Sands BE, Lewitzky S, et al. Secukinumab in Crohn's disease study group. Secukinumab, a human anti-IL-17A monoclonal antibody, for moderate to severe Crohn's disease: unexpected results of a randomised, double-blind placebo-controlled trial. Gut. 2012;61:1693-1700.

8. Targan SR, Feagan B, Vermeire S, et al. A randomized, doubleblind, placebo-controlled phase 2 study of brodalumab in patients with moderate-to-severe Crohn's disease. Am J Gastroenterol. 2016;111:1599-1607.

Publisher's Note Springer Nature remains neutral with regard to jurisdictional claims in published maps and institutional affiliations. 